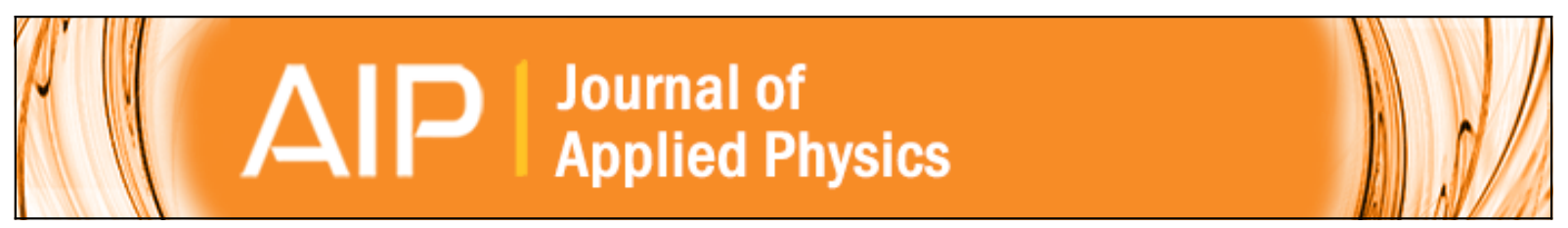

\title{
Effect of disorder on spin-transfer torque in magnetic tunnel junctions
}

Y.-H. Tang, Nicholas Kioussis, Alan Kalitsov, and Roberto Car

Citation: Journal of Applied Physics 109, 07 C920 (2011); doi: 10.1063/1.3565396

View online: http://dx.doi.org/10.1063/1.3565396

View Table of Contents: http://scitation.aip.org/content/aip/journal/jap/109/7?ver=pdfcov

Published by the AIP Publishing

\section{Articles you may be interested in}

Interfacial spin-filter assisted spin transfer torque effect in $\mathrm{Co} / \mathrm{BeO} / \mathrm{Co}$ magnetic tunnel junction

J. Appl. Phys. 117, 093901 (2015); 10.1063/1.4913780

Multi-orbit tight binding calculations for spin transfer torque in magnetic tunneling junctions

J. Appl. Phys. 111, 07 C904 (2012); 10.1063/1.3671773

Modeling of the spin-transfer torque switching in FePt/MgO-based perpendicular magnetic tunnel junctions: A combined ab initio and micromagnetic simulation study

Appl. Phys. Lett. 99, 032508 (2011); 10.1063/1.3615664

Effect of quantum confinement on spin transport and magnetization dynamics in dual barrier spin transfer torque magnetic tunnel junctions

J. Appl. Phys. 108, 104306 (2010); 10.1063/1.3503882

Spin-polarized current-induced torque in magnetic tunnel junctions

J. Appl. Phys. 99, 08G501 (2006); 10.1063/1.2151796

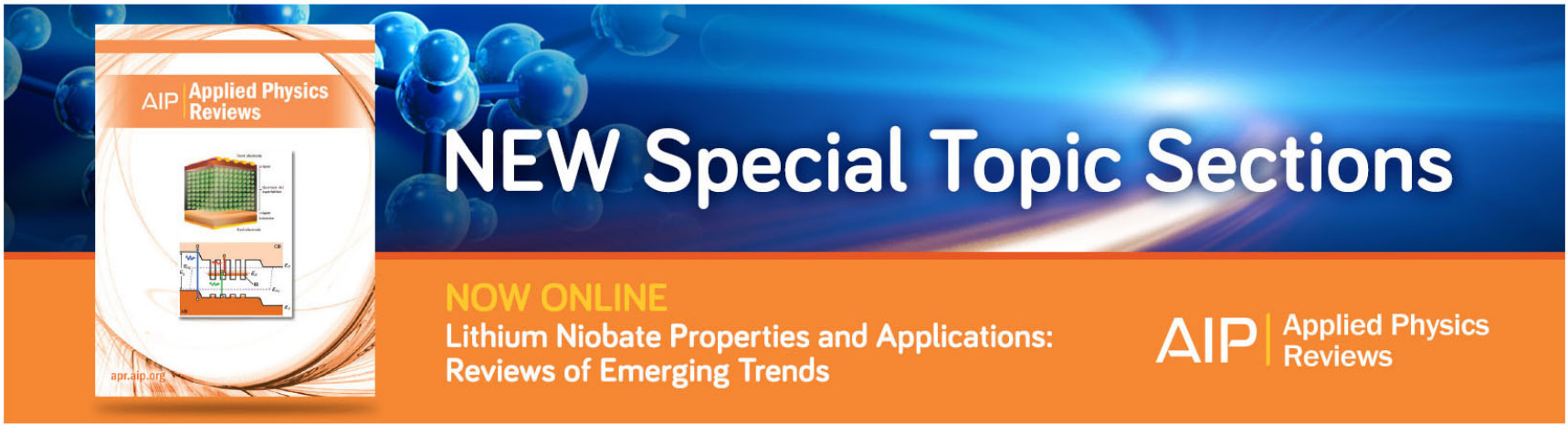




\title{
Effect of disorder on spin-transfer torque in magnetic tunnel junctions
}

\author{
Y.-H. Tang, ${ }^{1,2}$ Nicholas Kioussis, ${ }^{1, a)}$ Alan Kalitsov, ${ }^{1}$ and Roberto $\mathrm{Car}^{3}$ \\ ${ }^{1}$ Department of Physics, California State University, Northridge, California 91330-8268, USA \\ ${ }^{2}$ Department of Physics, National Central University, Jung-Li 32001, Taiwan \\ ${ }^{3}$ Department of Chemistry, Princeton University, Princeton, New Jersey 08544-0001, USA
}

(Presented 16 November 2010; received 25 September 2010; accepted 21 December 2010; published online 14 April 2011)

\begin{abstract}
We have generalized the nonequilibrium Green's functions Keldysh formalism to study the effect of interfacial disorder on the average spin transfer torque, $\left\langle T_{\|}\right\rangle$, in magnetic tunnel junctions (MTJs). We find a sinusoidal angular behavior of the average $\left\langle T_{\|}\right\rangle$as in ideal MTJs. We demonstrate for the first time that the general expression of the bias behavior of the average $\left\langle T_{\|}\right\rangle$in terms of the interplay of average spin current densities in collinear configurations is valid even in the presence of disorder. This explains the strong enhancement and sign reversal of $\left\langle T_{\|}\right\rangle$in the positive bias region, due to the disorder-induced resonance states at interface which selectively assist the transmission of right-coming electrons. (c) 2011 American Institute of Physics. [doi:10.1063/1.3565396]
\end{abstract}

\section{INTRODUCTION}

Noncollinear magnetic tunnel junctions (MTJ), where the magnetization of the left and right ferromagnetic (FM) leads, separated by a thin insulating barrier (IB), form an angle $\theta$, are of current interest. This is due to the recently discovered current-induced magnetization reversal ${ }^{1-3}$ via the so-called spin torque, which is relevant to a wide variety of applications in nonvolatile magnetic-random access memories and microwave oscillators. ${ }^{4-6}$

We have recently demonstrated that the bias dependence of spin-transfer torque component, $T_{\|}$, which is parallel to the plane of the magnetizations of the left and right FM leads, in both ideal ${ }^{7,8}$ and asymmetric ${ }^{9}$ MTJs has the general expression

$$
T_{\|}(\theta)=\frac{I_{z}^{(s)}(\mathrm{AF})-I_{z}^{(s)}(\mathrm{FM})}{2} \times \sin \theta
$$

in terms of the spin current density, $I_{z}^{(s)}[\mathrm{FM}(\mathrm{AF})]=\frac{\hbar}{2 e}$ $\left\{I^{\uparrow}[\mathrm{FM}(\mathrm{AF})]-I^{\downarrow}[\mathrm{FM}(\mathrm{AF})]\right\}$, of the FM and antiferromagnetic (AF) configurations. This suggests that the bias behavior of the noncollinear $T_{\|}(\theta)$ can be decomposed as the interplay between four independent nonequilibrium spinpolarized current densities, $I^{\sigma}$, solely in the FM and AF collinear configurations.

However, actual MTJs contain large amounts of disorder in the electrodes, in the barriers, and at the electrode/barrier interfaces. For example, both experimental ${ }^{10-12}$ and theoreti$\mathrm{cal}^{13}$ studies in MgO-based MTJs, have shown that oxygen vacancies and other structural defects inside the $\mathrm{MgO}$ barrier may strongly affect the tunnel magnetoresistance (TMR). On the other hand, there has been less attention of the effect of disorder on the bias dependence of the fieldlike, $T_{\perp},{ }^{14}$ and spin-transfer, $T_{\|},{ }^{15}$ components of the spin torque.

In this work, we employ the single-orbital simple-cubic tight-binding (TB) method and the nonequilibrium Keldysh formalism to understand the bias and angular dependence of

\footnotetext{
${ }^{a)}$ Electronic mail: nick.kioussis@csun.edu.
}

$T_{\|}$in the presence of disorder in MTJ. The treatment of charge- and spin-transport properties of disorder MTJ is a generalization of that introduced by Caroli et al. ${ }^{16}$ for nonmagnetic leads, based on an extension of the Keldysh formalism to the noncollinear configurations. ${ }^{8}$

\section{METHOD}

For a single impurity at $\left(\overrightarrow{\rho_{0}}, l\right)$, where $\rho_{0}$ and $l$ denote the impurity's coordinates parallel and perpendicular to the interface, respectively, the scattering potential is of the $\delta$-function form, ${ }^{17}$ i.e., $\hat{W}=\left[\varepsilon_{i m p} \delta(i-l)-\varepsilon_{b}\right] \hat{I} . \varepsilon_{i m p}\left(\varepsilon_{b}\right)$ is the impurity (IB) on-site energy, $i$ refers to an arbitrary layer inside IB, and $\hat{I}$ is the $2 \times 2$ unit matrix. The perturbed Keldysh Green's function $2 \times 2$ matrix in spin space, $\hat{\mathbb{G}}^{<}$, can be expressed as

$$
\begin{aligned}
\hat{\mathbb{G}}^{<}\left(\vec{\rho}, i ; \vec{\rho}^{\prime}, j\right)= & \hat{G}^{<}\left(\vec{\rho}, i ; \vec{\rho}^{\prime}, j\right) \\
& +\hat{G}^{<}\left(\vec{\rho}, i ; \overrightarrow{\rho_{0}}, l\right) \hat{\mathbb{T}}^{A} \hat{\tilde{G}}\left(\overrightarrow{\rho_{0}}, l ; \vec{\rho}^{\prime}, j\right) \\
& +\hat{G}\left(\vec{\rho}, i ; \overrightarrow{\rho_{0}}, l\right) \hat{\mathbb{T}}^{R} \hat{G}^{<}\left(\overrightarrow{\rho_{0}}, l ; \vec{\rho}^{\prime}, j\right) \\
& +\hat{G}\left(\vec{\rho}, i ; \overrightarrow{\rho_{0}}, l\right) \hat{\mathbb{T}}^{<} \hat{\tilde{G}}\left(\overrightarrow{\rho_{0}}, l, \vec{\rho}^{\prime}, j\right),
\end{aligned}
$$

where $\hat{G}, \hat{\tilde{G}}$ and $\hat{G}^{<}$are the $2 \times 2$ retarded, advanced, and Keldysh Green's function matrices in ideal MTJ, respectively. $i$ and $j$ denote the coordinates along the transport direction (y-axis), and $\vec{\rho}$, and $\vec{\rho}^{\prime}$ are two-dimensional vectors within the $\mathrm{x}-\mathrm{z}$ plane. The three $2 \times 2$ ' $\hat{\mathbb{T}}$ matrices are $\hat{\mathbb{T}}^{R}=\hat{W}$ $\left[I-\hat{W} \hat{G}\left(\overrightarrow{\rho_{0}}, l ; \vec{\rho}, l\right)\right]^{-1}, \hat{\mathbb{T}}^{A}=\hat{W}\left[I-\hat{W} \hat{\tilde{G}}\left(\overrightarrow{\rho_{0}}, l ; \overrightarrow{\rho_{0}}, l\right)\right]^{-1}$, and $\hat{\mathbb{T}}^{<}=\hat{\mathbb{T}}^{R} \hat{G}^{<}\left(\overrightarrow{\rho_{0}}, l ; \overrightarrow{\rho_{0}}, l\right) \hat{\mathbb{T}}^{A}$. The poles of these ${ }^{\mathbb{T}}$ matrices can give rise to the impurity-induced resonance states at the IB/FM interface, which may strongly affect not only the charge transfer property but also the spin torque effect. In addition, these impurity-induced resonance states can be tuned via external bias and the impurity on-site energy, leading to anomalous bias behaviors of both $I_{z}^{(s)}$ and $T_{\|}$.

Considering a layer of impurities with concentration, $c=N_{\text {imp }} / N$, where $N_{\text {imp }}$ and $N$ are the number of impurities 
and the total number of atomic sites per unit interfacial unit area, $\square$, the average of Eq. (2) can be determined by integrating over $\vec{\rho}$ and multiplying by $c$. Since we only consider the transport along the y-axis, one can set $\vec{\rho}=\vec{\rho}^{\prime}$, the average value of Eq. (2) can be recast in the form of

$$
\begin{aligned}
& \left\langle\hat{\mathbb{G}}^{<}\left(\vec{\rho}, i ; \vec{\rho}^{\prime}, j\right)\right\rangle \\
& \left.=\frac{1}{N} \sum_{k} \hat{G}_{k}^{<}(i, j)\right)+\frac{c}{N}\left\{\sum_{k} \hat{G}_{k}^{<}(i, l) \hat{\mathbb{T}}^{A} \hat{\tilde{G}}_{k}(l, j)\right. \\
& \left.+\sum_{k} \hat{G}_{k}(i, l) \hat{\mathbb{T}}^{R} \hat{G}_{k}^{<}(l, j)+\sum_{k} \hat{G}_{k}(i, l) \hat{\mathbb{T}}^{<} \hat{\tilde{G}}_{k}(l, j)\right\}
\end{aligned}
$$

In analogy with the ideal case, ${ }^{9}$ the average net $\left\langle T_{\|}\right\rangle$and $\left\langle I_{z}^{(s)}\right\rangle$ on the right FM lead per unit interfacial area, $\square$, in disorder MTJs can be simply expressed as the $\mathrm{x}$ and $\mathrm{z}$ components of the average spin current density at the right IB/FM interface, respectively, i.e.,

$$
\left\langle T_{\|}\right\rangle=\frac{e t}{16 \pi^{3} \hbar} \int \operatorname{Tr}\left[\left(\left\langle\hat{\mathbb{G}}_{\alpha^{\prime} b}^{<}\right\rangle-\left\langle\hat{\mathbb{G}}_{b \alpha^{\prime}}^{<}\right\rangle\right) \sigma_{x}\right] d E d \mathbf{k}_{\|}
$$

and

$$
\left\langle I_{z}^{(s)}\right\rangle=\frac{-e t}{16 \pi^{3} \hbar} \int \operatorname{Tr}\left[\left(\left\langle\hat{\mathbb{G}}_{\alpha^{\prime} b}^{<}\right\rangle-\left\langle\hat{\mathbb{G}}_{b \alpha^{\prime}}^{<}\right\rangle\right) \sigma_{z}\right] d E d \mathbf{k}_{\|} .
$$

Here, $\alpha^{\prime}$ is the first site of the right FM lead, b is the last site in the IB, $\sigma=\left(\sigma_{x}, \sigma_{y}, \sigma_{z}\right)$ is the vector of the $2 \times 2$ Pauli matrix, $\mathbf{k}_{\|}$is the transverse component of the wave vector, and the energy integral is over occupied states.

In this study, we consider the case of a layer of nonmagnetic impurities placed in the last IB layer next to the right IB/FM interface, i.e., $l=b$, with $\varepsilon_{i m p}=0.0 \mathrm{eV}$, where the Fermi energy is set to zero. We apply the same TB energy parameters used in Ref. 9, which provides a realistic choice for systems based on magnetic transition metals and their alloys ${ }^{8}$ and gave an excellent agreement for the bias dependence of both $T_{\|}$and $T_{\perp}$ between our previous theoretical predictions $^{7}$ and experiment. ${ }^{18}$

\section{RESULTS AND DISCUSSION}

In Fig. 1, we present the bias behaviors of $\left\langle I_{z}^{(s)}(\mathrm{FM})\right\rangle$ (black solid curve) and $\left\langle I_{z}^{(s)}(\mathrm{AF})\right\rangle$ (gray solid curve) for

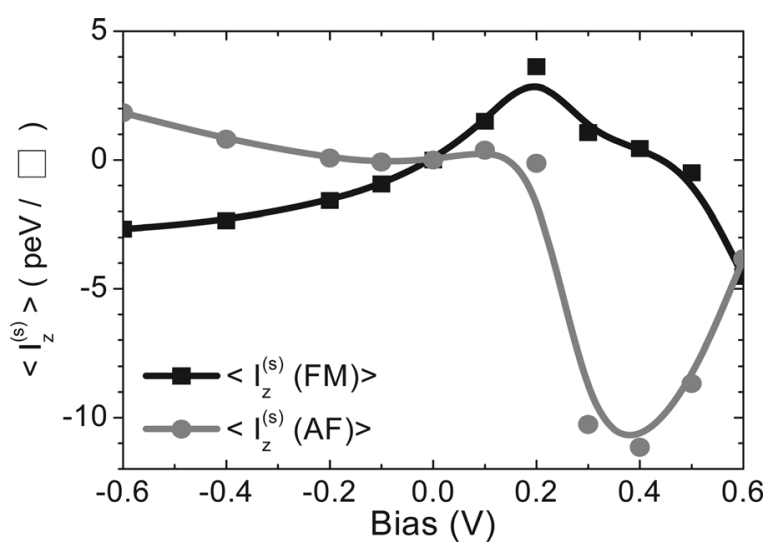

FIG. 1. Bias dependence of $\left\langle I_{z}^{(s)}(\mathrm{FM})\right\rangle$ (black solid curve) and $\left\langle I_{z}^{(s)}(\mathrm{AF})\right\rangle$ (gray solid curve) for disorder cases with $\theta=\pi / 2$ and $\varepsilon_{i m p}=0.0 \mathrm{eV}$.

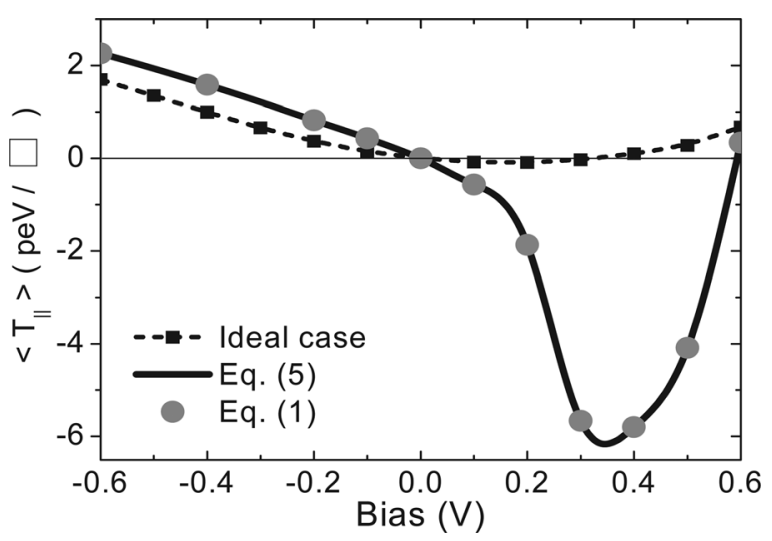

FIG. 2. Bias dependence of $T_{\|}(\theta / 2)$ (black dashed line) for the ideal case and $\left\langle T_{\|}(\theta / 2)\right\rangle$ (black solid line) for the disorder case calculated by Eq. (1) in (Ref. 9) and Eq. (5), respectively. The gray solid circles represent $\left\langle T_{\|}(\theta / 2)\right\rangle$ for the disorder case calculated by Eq. (1) with $\theta=\pi / 2$.

disorder cases with $\theta=\pi / 2$ and $\varepsilon_{i m p}=0.0 \mathrm{eV}$. Unlike the linear and quadratic bias dependence of $I_{z}^{s}$ in ideal MTJs (Figs. 4(b) and 4(c) of Ref. 9), $\left\langle I_{z}^{(s)}(\mathrm{FM})\right\rangle^{2}$ and $\left\langle I_{z}^{(s)}(\mathrm{AF})\right\rangle$ both exhibit very distinct bias behaviors especially in the positive bias region. This is due to the fact that under positive bias (electrons flow from the right to the left FM lead), the transmission of the right-flowing electrons is strongly affected by the impurity-induced resonance states at the right IB/FM interface. On the other hand, in the negative bias region, the smaller contribution from the impurity-assisted right-flowing electrons leads to similar bias behavior in ideal case.

In Fig. 2, the bias dependence of $T_{\|}(\theta / 2)$ for the ideal case calculated from Eq. (1) in Ref. 9 and $\left\langle T_{\|}(\theta / 2)\right\rangle$ for disorder case calculated by Eq. (5) are displayed in black dashed and solid lines, respectively. The gray solid circles are calculated from Eq. (1) with $\theta=\pi / 2$ for disorder case. The excellent agreement between the black solid lines and gray solid circles indicates that the general Eq. (1) is valid. In Fig. 3, we present the angular dependence of $\left\langle T_{\|}\right\rangle$in disorder case under bias (a) $-0.2 \mathrm{~V}$ and (b) $0.2 \mathrm{~V}$. Similar to the ideal MTJ, the sinusoidal angular behavior still holds, which is in agreement with Birol's results at low spin polarization. ${ }^{15}$ Therefore, our numerical results demonstrate that the general expression

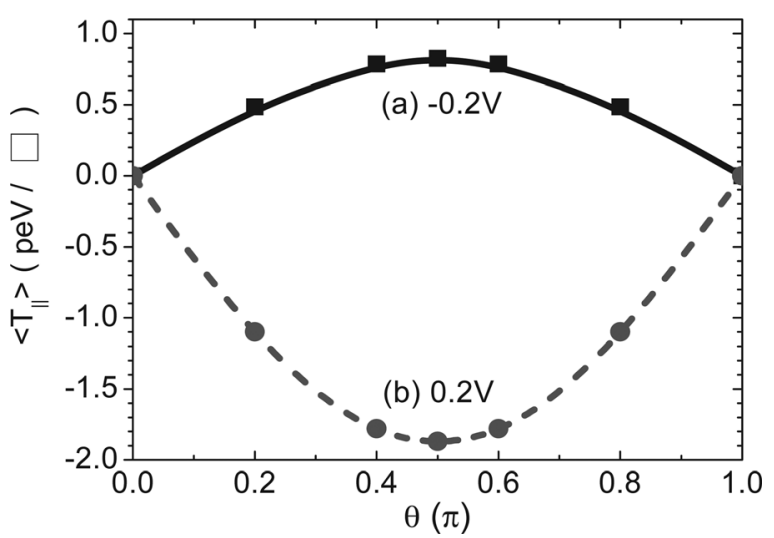

FIG. 3. The angular dependence of $\left\langle T_{\|}\right\rangle$in disorder cases under bias (a) $\mathrm{V}=-0.2 \mathrm{~V}$ and (b) $\mathrm{V}=0.2 \mathrm{~V} . \theta$ is the angle between the magnetizations of two FM leads. 
Eq. (1) is valid even in the presence of disorder and hence the bias behavior of $\left\langle T_{\|}\right\rangle$simply reduces to the interplay of the average spin current densities in the collinear configurations.

In addition, as shown in Fig. 2, the strong enhancement and the sign reversal of $\left\langle T_{\|}(\theta / 2)\right\rangle$ only in the positive bias region, results from the disorder-induced resonance states at the right FM/IB interface. This gives rise to a disorder-induced enhancement of $\left\langle T_{\|}(\theta / 2)\right\rangle$ at $0.3 \mathrm{~V}$ by a factor of about 50 compared to the ideal MTJ (Fig. 4(a) of Ref. 9). In contrast, in the negative bias regime the magnitude and bias behavior of $\left\langle T_{\|}(\theta / 2)\right\rangle$ almost remain the same as in the ideal case.

\section{CONCLUSION}

In conclusion, we present an implementation of the generalized Keldysh Green's function method to calculate the angular and bias dependence of the average spin transfer torque, $\left\langle T_{\|}\right\rangle$, for a layer of nonmagnetic impurities at the right IB/FM interface of the MTJ. We find that $\left\langle T_{\|}\right\rangle$has a sinusoidal bias behavior as the ideal case. Our numerical results demonstrate that the general expression Eq. (1), is valid even in the presence of disorder and hence the bias behavior of $\left\langle T_{\|}\right\rangle$simply reduces to the interplay of the average spin current densities in the collinear configurations. The disorderinduced resonance states at the right IB/FM interface yield a strong enhancement including a sign reversal for both $\left\langle I_{z}^{s}\right\rangle$ and $\left\langle T_{\|}\right\rangle$in the positive bias region, due to the larger contribution from the right-coming electrons.

\section{ACKNOWLEDGMENTS}

We acknowledge support from the National Center for Theoretical Sciences (South), Taiwan. The work at California
State University, Northridge was supported by NSF-PREM Grant No. DMR-00116566 and at Princeton University by DOE Grant No. DE-FG02-05ER46201.

${ }^{1}$ C. Chappert, A. Fert, and F. N. V. Dau, Nat. Matter. 6, 813 (2007).

${ }^{2}$ Y. Huai, F. Albert, P. Nguyen, M. Pakala, and T. Valet, Appl. Phys. Lett. 84, 3118 (2004).

${ }^{3}$ G. D. Fuchs, N. C. Emley, I. N. Krivorotov, P. M. Braganca, E. M. Ryan, S. I. Kiselev, J. C. Sankey, D. C. Ralph, and R. A. Buhrman, Appl. Phys. Lett. 85, 1205 (2004).

${ }^{4}$ S. Parkin, J. Xin, C. Kaiser, A. Panchula, K. Roche, and M. Samant, Proc. IEEE 91, 661 (2003).

${ }^{5}$ S. I. Kiselev, J. C. Sankey, I. N. Krivorotov, N. C. Emley, R. J. Schoelkopf, R. A. Buhrman, and D. C. Ralph, Nature 425, 380 (2003).

${ }^{6}$ S. Kaka, M. R. Pufall, H. Rippard, T. J. Silva, S. E. Russek, and J. A. Katine, Nature 437, 389 (2005).

${ }^{7}$ I. Theodonis, N. Kioussis, A. Kalitsov, M. Chshiev, and W. H. Butler, Phys. Rev. Lett. 97, 237205 (2006).

${ }^{8}$ A. Kalitsov, M. Chshiev, I. Theodonis, N. Kioussis, and W. H. Butler, Phys. Rev. B 79, 174416 (2009).

${ }^{9}$ Y.-H. Tang, N. Kioussis, A. Kalitsov, W. H. Butler, and R. Car, Phys. Rev. B 81, 054437 (2010).

${ }^{10}$ S. Yuasa, T. Nagahama, A. Fukushima, Y. Suzuki, and K. Ando, Nat. Mater. 3, 868 (2004).

${ }^{11}$ P. G. Mather, J. C. Read, and R. A. Buhrman, Phys. Rev. B 73, 205412 (2006).

${ }^{12}$ G. X. Miao, Y. J. Park, J. S. Moodera, M. Seibt, G. Eilers, and M. Münzenberg, Phys. Rev. Lett. 100, 246803 (2008).

${ }^{13}$ J. P. Velev, K. D. Belashchenko, S. S. Jaswal, and E. Y. Tsymbal, Appl. Phys. Lett. 90, 072502 (2007).

${ }^{14}$ M. Ye. Zhuravlev, E. Y. Tsymbal, and A. V. Vedyayev, Phys. Rev. Lett. 94, 026806 (2005).

${ }^{15}$ T. Birol and P. W. Brouwer, Phys. Rev. B. 80, 014434 (2009).

${ }^{16}$ R. Combescot, J. Phys. C: Solid St. Phys. 4, 2611 (1971).

${ }^{17}$ A. Kalitsov, A. Coho, N. Kioussis, A. Vedyayev, M. Chshiev, and A. Granovsky, Phys. Rev. Lett. 93, 046603 (2004).

${ }^{18}$ H. Kubota, A. Fukushima, K. Yakushiji, T. Nagahama, S. Yuasa, K. Ando, H. Maehara, Y. Nagamine, K. Tsunekawa, D. D. Djayaprawira, N. Watanabe, and Y. Suzuki, Nature Phys. 4, 37 (2008). 